EPJ Web of Conferences 52, 10003 (2013)

DOI: $10.1051 /$ epjconf/20135210003

(C) Owned by the authors, published by EDP Sciences, 2013

\title{
Search for high-energy gamma-ray emission and upgrade of the GAMMA experiment on Mt. Aragats
}

\author{
R.M. Martirosov ${ }^{1, a, b}$, H. Babayan², A.D. Erlykin ${ }^{3}$, Y.A. Gallant ${ }^{4}$, A.P. Garyaka ${ }^{1}$, L.W. Jones ${ }^{5}$, J. Kempa ${ }^{6}$, N.M. \\ Nikolskaya $^{3}$, B. Pattison ${ }^{7}$, J. Procureur ${ }^{8}$, Ch. Spiering ${ }^{9}$, S.V. Ter-Antonyan ${ }^{10}$, and H. Vardanyan ${ }^{1}$ \\ ${ }^{1}$ Alikhanyan National Laboratory, Yerevan, Armenia \\ ${ }^{2}$ State Engineering University of Armenia, Yerevan, Armenia \\ ${ }^{3}$ P.N. Lebedev Physical Institute of the RAS, Moscow, Russia \\ ${ }^{4}$ LUPM, Université Montpellier 2, CNRS/IN2P3, Montpellier, France \\ ${ }^{5}$ Department of Physics, University of Michigan, Ann Arbor, USA \\ ${ }^{6}$ Warsaw University of Technology, Branch in Plock, Poland \\ ${ }^{7}$ CERN, Geneva, Switzerland \\ ${ }^{8}$ Centre d'Études Nucléaires de Bordeaux-Gradignan, Gradignan, France \\ ${ }^{9}$ DESY, Zeuthen, Germany \\ ${ }^{10}$ Department of Physics, Southern University, Baton Rouge, USA
}

\begin{abstract}
We present the current status of the search for possible diffuse or localized gamma-ray emission at energies above about $100 \mathrm{TeV}$ with the GAMMA air shower detector which is located at $3200 \mathrm{~m}$ a.s.l. on Mt. Aragats, Armenia. For this search we select muon-poor extensive air showers detected by GAMMA. We discuss ongoing studies of improved criteria for the selection of muon-poor showers, taking into account the geometry of the muon underground detector array in 2004-2011. A modernization of the muon array was completed between October 2011 and February 2012. Sixty additional muon scintillation detectors were installed and are now taking data. They will improve the selection of muon-poor showers, the primary energy estimation, and should also yield improved information on the mass composition.
\end{abstract}

\section{Introduction}

One of the main challenges in the energy region of the knee of the primary cosmic ray spectrum is the search for and investigation of primary $\gamma$-rays, including a study of the diffuse flux. The flux of ultra high energy (UHE) $\gamma$ rays is extremely small. This is why it is practically impossible to register them with facilities installed on balloons or satellites. At the same time, crossing the Earth's atmosphere UHE $\gamma$-rays generate electromagnetic cascades, which are similar to the electromagnetic component of extensive air showers (EAS) generated by cosmic rays. Therefore the methods to register cascades generated by UHE $\gamma$-rays are adopted from those of experiments on EAS studies.

The study of diffuse $\gamma$-rays at energies greater than 100 $\mathrm{TeV}$ is - almost exclusively - based on the registration and selection of EAS with an abnormally small relative content of muons. This approach was proposed in 1960 by Maze and Zawadzki [1].

The selection of showers initiated by very high energy primary photons $\left(\geq 10^{14} \mathrm{eV}\right)$ requires the measurement of a maximum number of shower parameters, $X_{i}$, each

\footnotetext{
a corresponding author

be-mail: romenmartirosov@ rambler.ru
}

with the best possible resolution $\left[\sigma\left(X_{i}\right) /\left\langle X_{i}\right\rangle\right]_{\text {rec }}$. Taken together, these shower parameters - like $\rho_{\mu}, N_{\mu}$ - should have enough selection power to identify $\gamma$-EAS muon components. The resolution of the parameters improves with increasing effective area with respect to the measured component of the EAS. Therefore the recent upgrade of the GAMMA experiment with 60 additional detectors for the muon hodoscope is important for obtaining more reliable information on the EAS generated by primary $\gamma$-rays.

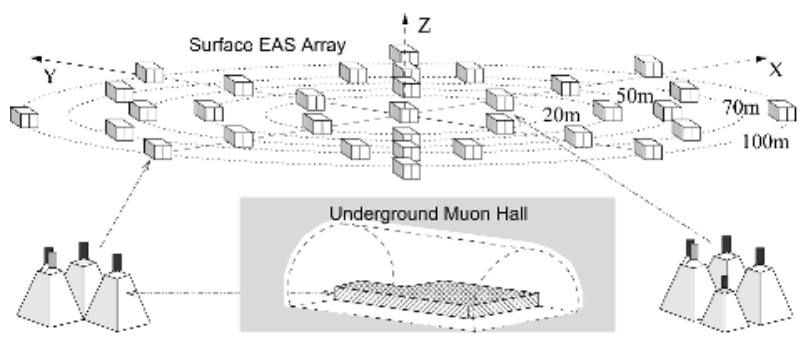

Figure 1. Layout of the GAMMA experiment. 


\section{The GAMMA experiment}

The GAMMA array is located on Mt. Aragats in Armenia $\left(3200 \mathrm{~m}\right.$ a.s.l., $\left.700 \mathrm{~g} / \mathrm{cm}^{2}\right)$ and consisted, until October 2011, of 116 surface and 150 underground scintillation detectors [2,3] (Fig. 1). GAMMA is able to measure the electromagnetic (by surface detectors) and muon components (by the underground muon detectors) of cosmic-ray EAS with energy $10^{14}-10^{17} \mathrm{eV}$. The muon energy threshold is $5 \mathrm{GeV}$. The investigation of the characteristics of these two components, expressed by a number of EAS observables, provides a basis for multi-parameter analyses and allows the collection of reliable experimental data on the main characteristics of the primary cosmic rays and $\gamma$-rays in the above energy range.

\section{Improved selection of $\mu$-poor showers}

We already published an all-sky upper limit on diffuse $\gamma$ ray emission [4], but more detailed investigations need to be performed.

In our earlier study [4], events were selected based on the reconstructed shower core position $(X, Y)$ at ground level $(Z=0)$, with a selection radius, $R$, relative to the center of the installation (about which the surface detectors are symmetrically distributed). The events used in [4] were selected by the shower core position and zenith angle with the criteria:

$$
R<15 \mathrm{~m} \quad \text { and } \quad \theta<30^{\circ} .
$$

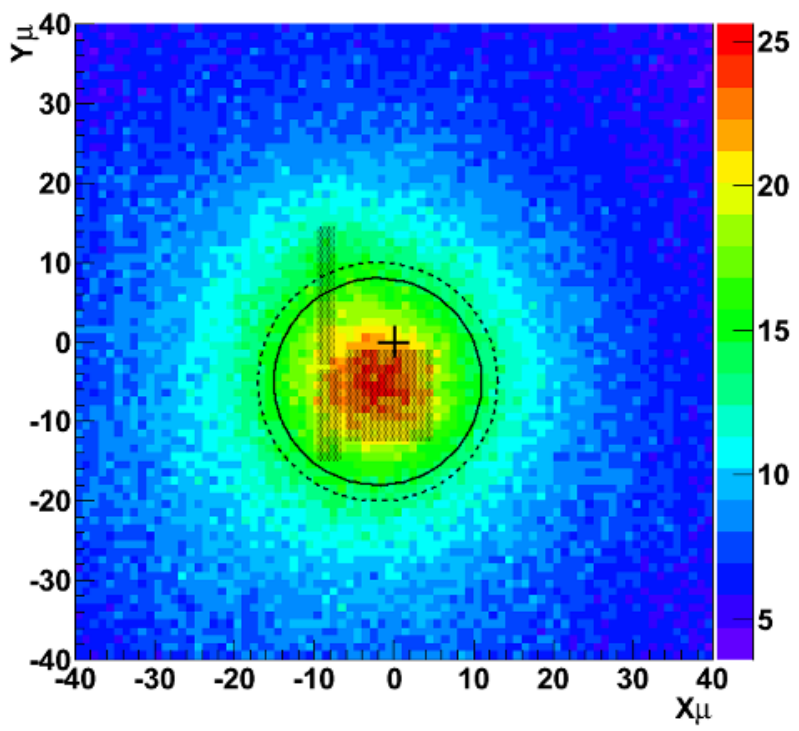

Figure 2. Distribution of the average number of fired muon detectors $\left\langle N_{\mu, \mathrm{f}}\right\rangle$ as a function of the shower axis position $\left(X_{\mu}, Y_{\mu}\right)$ in the muon detector plane, from 2008 GAMMA data. The shaded area depicts the locations of the muon detectors prior to the upgrade, while the cross shows the geometrical center of the GAMMA installation. The shower selection areas $R_{\mu}<15 \mathrm{~m}$ and $R_{\mu}<13 \mathrm{~m}$ discussed in the text are shown with dashed and solid circles, respectively.
In order to improve the selection power for muon-poor showers, we more recently studied the detected muon content of showers as a function of their extrapolated shower core position $\left(X_{\mu}, Y_{\mu}\right)$ at the level of the underground muon detector hall $(Z=-13.98 \mathrm{~m})$. Figure 2 shows the twodimensional distribution of the average number of fired muon detectors $\left\langle N_{\mu, \mathrm{f}}\right\rangle$ as a function of $X_{\mu}$ and $Y_{\mu}$, for GAMMA events recorded in 2008. It can be seen that $\left\langle N_{\mu, \mathrm{f}}\right\rangle$ is highest in a region around the centroid of the muon detectors at that time (prior to the GAMMA upgrade), and displaced from the center of the installation and of the surface detectors (shown as a cross). This asymmetry is due to the relative lack of muon detectors close to the shower core when $Y_{\mu}$ is positive, which has now been mitigated by the GAMMA upgrade.

As any contribution from electromagnetic showers initiated by $\gamma$-rays must be very small, figure 2 illustrates the average detected muon content of hadronic showers, i.e. showers initiated by hadronic primary cosmic rays. It suggests that hadronic showers with higher $\left\langle N_{\mu, \mathrm{f}}\right\rangle$ (and thus better separated from muon-poor $\gamma$-ray showers) can be selected with a shower core position criterion in the muon detector plane, and relative to the centroid of the muon detector locations (prior to the upgrade). We denote by $R_{\mu}$ the shower core distance relative to the approximate centroid $\left(X_{\mu}, Y_{\mu}\right)=(-2 \mathrm{~m},-5 \mathrm{~m})$, and examine two sets of core position and zenith angle criteria, namely

$$
R_{\mu}<15 \mathrm{~m} \quad \text { and } \quad \theta<30^{\circ},
$$

denoted by blue curves in figures 3 and 4, and

$$
R_{\mu}<13 \mathrm{~m} \quad \text { and } \quad \theta<45^{\circ},
$$

denoted by red curves. The corresponding shower core selection regions are shown in figure 2 as dashed and solid

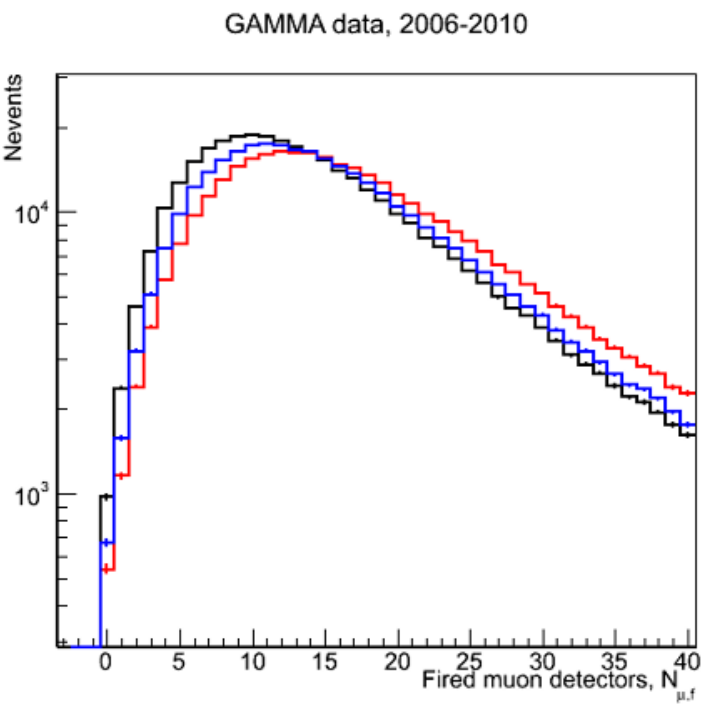

Figure 3. Histogram of the number of fired muon detectors $N_{\mu, \mathrm{f}}$ for GAMMA events from 2006-2010, for the three different selection criteria (1), (2) and (3) discussed in the text, respectively shown as black, blue and red lines. 
GAMMA data, 2006-2010

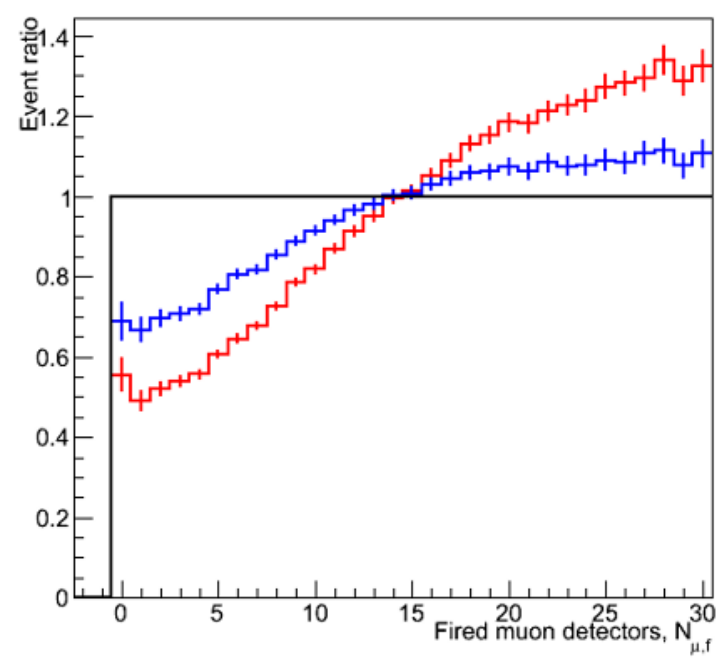

Figure 4. Ratio of the histograms shown in figure 3 for the selection criteria (2) and (3), shown as blue and red lines respectively, to the histogram with the original selection criteria (1), illustrated with a black line.

circles, respectively. Figure 3 shows the corresponding distributions in the number of fired muon detectors, $N_{\mu, \mathrm{f}}$, for GAMMA events from 2006-2010. For all three sets of selection criteria (1), (2) and (3), the following additional shower selection criteria were also applied:

$$
\begin{aligned}
\text { Shower size: } & N_{e}>10^{5} \\
\text { Shower age: } & 0.4<s<1.5 \\
\text { Fit quality: } & \text { red. } \chi^{2}<2.5
\end{aligned}
$$

The three sets of selection criteria retain a similar total number of events, enabling a straightforward comparison. It can be seen in figure 3 that the selection criteria (2), and even more so (3), result in a larger average $N_{\mu, \mathrm{f}}$ and a lower number of muon-poor showers than the original criteria (1).

This reduction in muon-poor showers is shown more quantitatively in figure 4 , which shows the ratio of the histograms with the criteria (2) and (3) to that with the original criteria (1). It can be seen that at low muon numbers, $N_{\mu, \mathrm{f}}$, the criteria (2) effect a reduction tending towards a factor $\sim 0.7$, and a factor $\sim 0.5$ with the criteria (3), relative to the original criteria (1). This reduction in muon-poor hadronic showers implies an improved separation from any $\gamma$-ray showers which might be present. The lower reduction observed in the $N_{\mu, \mathrm{f}}=0$ bin is, however, not statistically significant. Nonetheless, the separation power of such criteria is promising to improve the sensitivity of searches for $\gamma$-ray sources in specific sky regions. We are currently working towards validation of this approach using $\gamma$-ray and hadronic shower simulations.

\section{Upgrade of the muon detector array}

During October 2011 - February 2012 the underground muon detector array has been upgraded. Sixty additional

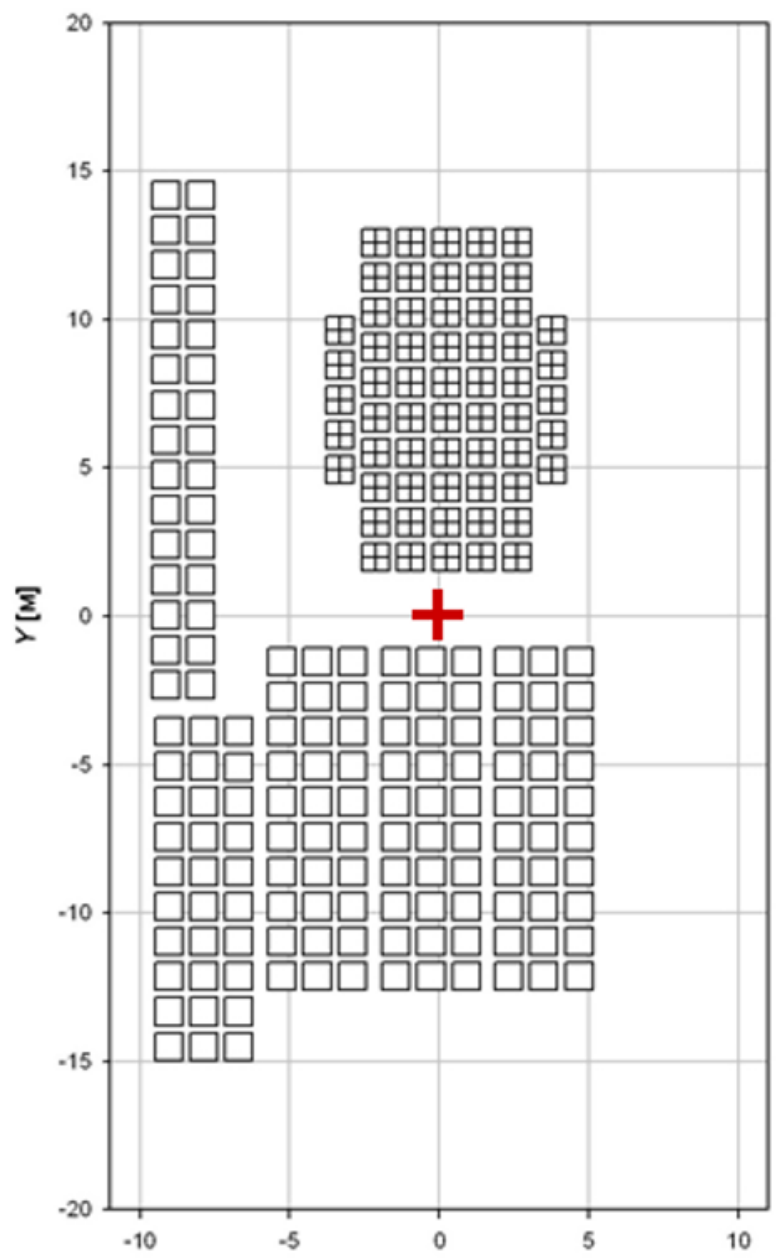

Figure 5. Arrangement of the muon detectors: crossed squares 60 new detectors.

scintillation detectors were installed. The new configuration of the muon array is presented in Fig. 5. Besides increasing the total number of muon detectors up to 210 , it can be seen that the arrangement of the muon detectors is now more symmetrical. This should certainly improve the separation between hadronic and any muon-poor gammaray showers and/or increase the effective selection area where we can achieve separation as before. It should also improve the muon size reconstruction for hadronic cosmic ray composition studies.

\section{Acknowledgements}

This work has been partly supported by the research grant no. 11-1c 345 from the Armenian Government, by the Moscow P.N. Lebedev Institute, DESY, the CNRSSCS exchange grant no. 24947 and the "Hayastan" AllArmenian Fund.

\section{References}

[1] R.Maze and A.Zawadzki, Nuovo Cimento, 17, 625 (1960) 
[2] A.P. Garyaka, R.M. Martirosov, S.V. Ter-Antonyan, N.M. Nikolskaya, Y.A. Gallant, L.W. Jones and J. Procureur, Astroparticle Physics 28, 169 (2007)

[3] A.P. Garyaka, R.M. Martirosov, S.V. Ter-Antonyan, A.D. Erlykin, N.M. Nikolskaya, Y.A. Gallant, L.W. Jones and J. Procureur, J. Phys. G: Nucl. Part. Phys.
35, 115201 (2008)

[4] Romen M. Martirosov, Samvel V. Ter-Antonyan, Anatoly D. Erlykin, Alexandr P. Garyaka, Natalya M. Nikolskaya, Yves A. Gallant and Lawrence W. Jones, Proc. of the 31st ICRC, Łódź (arXiv:0905.3593 [astroph]) (2009). 